\title{
Highly nonlinear solitary wave propagation in Y-shaped granular crystals with variable branch angles
}

\author{
D. Ngo, ${ }^{1, *}$ F. Fraternali, ${ }^{2}$ and C. Daraio ${ }^{1, \dagger}$ \\ ${ }^{1}$ Engineering and Applied Science, California Institute of Technology, Pasadena California 91125, USA \\ ${ }^{2}$ Department of Civil Engineering, University of Salerno, Fisciano (SA), Italy
}

(Received 12 August 2011; published 6 March 2012)

\begin{abstract}
We study the propagation of highly nonlinear waves in a branched (Y-shaped) granular crystal composed of chains of spherical particles of different materials, arranged at variable branch angles. We experimentally test the dynamic behavior of a solitary pulse, or of a train of solitary waves, crossing the Y-junction interface, and splitting between the two branches. We describe the dependence of the split pulses' speed and amplitude on the branch angles. Analytic predictions based on the quasiparticle model and numerical simulations based on Hertzian interactions between the particles are found to be in excellent agreement with the experimental data.
\end{abstract}

DOI: 10.1103/PhysRevE.85.036602 PACS number(s): 43.25.+y, 45.70.-n, 05.45.Yv, 46.40.Cd

\section{INTRODUCTION}

Granular crystals are systems composed of particles in contact with each other, arranged in specific geometrical configurations. These systems are characterized by a highly nonlinear dynamic response derived from the Hertzian contact interaction between particles and from the zero tensile resistance between grains. One-dimensional (1D) granular crystals have been studied extensively [1-20]. When composed of uniform particles, they have been shown to support the propagation of highly nonlinear solitary waves characterized by a compact shape and a traveling speed dependent on the wave amplitude [1,2]. The dynamic response of granular crystals can be controlled by varying the particle's material properties $[3,12,13]$ or by introducing defects or interfaces $[4,10,15,16]$. In addition, the presence of static precompression can tune the systems in the linear, weakly nonlinear or highly nonlinear dynamic regime $[1,3,13]$. Understanding the dynamic response of uniform and heterogeneous granular crystals provides fundamental insight on the propagation of acoustic signals in highly packed granular media (e.g., soil, sand, etc.) $[1,18,19]$. Granular crystals also have been proposed for a variety of engineering applications, including energy trapping and impulse disintegration $[8,10,15,16,20]$, nondestructive evaluation $[6,21]$, and sound focusing devices [22]. Few attempts have been made to study the propagation of highly solitary waves in two-dimensional (2D) systems [23-27]. In particular, systems composed of spherical particles arranged in a symmetric Y-shaped configuration at fixed branch angles also have been studied [24-26]. The study of the dynamics of branched systems showed that highly nonlinear solitary waves can follow a bent trajectory, and they can split when crossing a $\mathrm{Y}$ junction. The propagation properties of a single solitary wave in Y-shaped systems composed of spherical particles of the same material also was found to depend on the branch angles of the system [27]. Recently, Y-shaped granular crystals have been proposed to realize acoustic logic gates [28].

${ }^{*}$ Current address: School of Engineering, Eastern International University, Binh Duong, Vietnam.

${ }^{\dagger}$ daraio@caltech.edu
In this paper, we extend the previous studies on Y-shaped systems by performing analytic, numerical, and experimental studies on the propagation of a single solitary wave or of a train of solitary waves in both uniform and nonuniform systems with variable angles between the two branches. We investigate the effect of the system geometry (varying the branch angles) on the tunability of solitary waves' speed and amplitude. The outcomes of the present paper are promising for the design of composite materials capable of redirecting stresses and impulses and for understanding the angular dependence of dynamic load transfer in 2D granular material media [24].

The paper is organized as follows: In Sec. II, we describe the experimental setup. In Sec. III, we present the quasiparticle approach used to analyze the solitary wave propagation in the system. In Sec. IV, we present the results and a detailed discussion. We end the paper with conclusions, and we include an Appendix describing the discrete element model used to simulate the solitary wave propagation in the system.

\section{EXPERIMENTAL SETUP}

We designed a set of Y-shaped support guides with different geometries, composed of one main stem and two branches as shown in Fig. 1 [27] where $\alpha$ is the branch angle between the first branch and the main stem and $\beta$ is the branch angle between the second branch and the main stem. The set includes both symmetric guides $(\alpha=\beta)$ and asymmetric guides $(\alpha \neq \beta)$. All the guides were made from polycarbonate and were filled with chains of stainless steel (type 440C) or aluminum spherical particles of uniform diameter (equal to $9.52 \mathrm{~mm}$ ). Each one of the main stem and the two branches contained 15 particles. At the interface, the three chains (the main stem and the two branches) shared one particle. The properties of the materials of the particles and the guide used in this paper are summarized in Table I. In order to monitor the wave propagation and to calculate the wave speed before and after the Y-junction interface, we placed six instrumented particles at selected positions in the system (two sensors in main stem and two in each branch). The instrumented particles were custom fabricated in our laboratory by introducing a piezoelectric disk (provided by Steiner \& Martins, Inc.) with custom wiring between two 
(a)

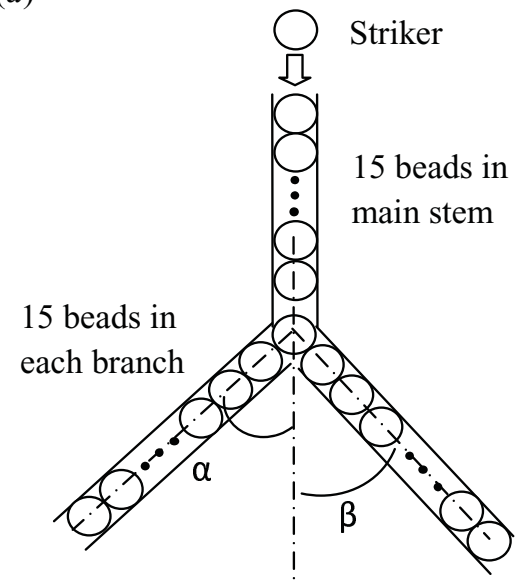

(b)

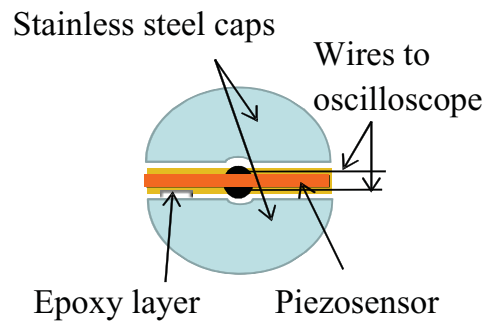

FIG. 1. (Color online) (a) Experimental setup showing the Y-shaped guide and indicating the number of beads composing each portion of the system. (b) Schematic of the composition of a particle with an embedded piezogauge [12-14]. halves of a spherical particle. The final assembly of the sensor particles was achieved following a procedure similar to the one described in Refs. [12-14] [see Fig. 1(b)]. All the sensors were precalibrated by assuming conservation of linear momentum to obtain the relation between voltage output obtained from the oscilloscope and the corresponding force experienced by the particle. These instrumented particles were connected to Tektronix oscilloscopes for data acquisition. The whole setup was tilted at a small angle $\left(\sim 4^{\circ}\right)$ to ensure contact among all particles. Single solitary waves propagating in the system were generated by impacting a striker particle, identical to the other particles in the chain, on the first particle of the main stem. Trains of solitary waves were created by using a striker with larger mass. The striker's impact velocity was measured using an optical velocimeter connected to a digital oscilloscope.

\section{QUASIPARTICLE MODEL}

We employed a simple quasiparticle model to explain the dependence of the single solitary waves' speed and amplitude on the branch angles in uniform Y-shaped systems.

\section{A. Quasiparticle model}

The quasiparticle analogy to analyze the propagation of highly nonlinear solitary waves in an uncompressed 1D uniform chain of spherical particles was suggested by Nesterenko [1]. Following this approach, the energy and momentum carried by a single solitary wave can be considered equivalently to the energy and momentum carried by a particle having an effective mass $m_{\text {eff }}$ and moving with an effective speed $V_{\text {eff }}$. Job et al. [16] used this quasiparticle approach to obtain a

TABLE I. Properties of the materials composing the highly nonlinear systems tested experimentally [29].

\begin{tabular}{lcccc}
\hline \hline & $\begin{array}{c}\text { Elastic modulus } \\
{[\mathrm{GPa}]}\end{array}$ & $\begin{array}{c}\text { Poisson } \\
\text { ratio }\end{array}$ & $\begin{array}{c}\text { Density } \\
{\left[\mathrm{kg} / \mathrm{m}^{3}\right]}\end{array}$ & $\begin{array}{c}\text { Bead mass } \\
{[\mathrm{g}]}\end{array}$ \\
\hline Stainless steel beads & 200 & 0.28 & 7800 & 3.5 \\
Aluminum beads & 69 & 0.3 & 2740 & 1.2 \\
Polycarbonate (wall) & 2.4 & 0.37 & 1220 & \\
\hline \hline
\end{tabular}

quantitative analysis of the formation of solitary wave trains in a horizontal stepped chain when a single solitary wave propagated from a chain composed of large spherical particles to a chain composed of small spherical particles. The relations of the effective mass and speed of the quasiparticle to the dynamic properties of a traveling single solitary wave and to the material properties of the particles in the chain can be obtained as [16]

$$
\begin{aligned}
& m_{\text {eff }}=1.345 m, \\
& V_{\text {eff }}=1.385 V_{m},
\end{aligned}
$$

where $m$ is the mass of a particle in the chain and $V_{m}$ is the maximum particle velocity. $V_{m}$ is related to the solitary wave speed $V_{s w}$ and the maximum strain $\xi_{m}$ in the chain by [1]

$$
V_{m}=V_{s w} \xi_{m} .
$$

The maximum strain in the chain can be expressed as a function of the solitary wave speed and material properties of the particles as [1]

$$
\xi_{m}=\left(\frac{\sqrt{5}}{2}\right)^{4} \frac{1}{c^{4}} V_{s w}^{4},
$$

where $c=\sqrt{\frac{2 E}{\pi \rho_{0}\left(1-v^{2}\right)}}$, with $E$ being the elastic modulus of the particles, $\rho_{0}$ being the particle's density, and $v$ being the Poisson ratio. By substituting Eqs. (3) and (4) into Eq. (2), one finds the relation between the quasiparticle's effective speed and solitary wave speed as

$$
V_{\mathrm{eff}}=1.385\left(\frac{\sqrt{5}}{2}\right)^{4} \frac{1}{c^{4}} V_{s w}^{5} \text {. }
$$

\section{B. Uniform Y-shaped system}

Using the quasiparticle approach, we analyze the transmissions of energy and momentum carried by the single solitary wave in the main stem to the two branches in a Y-shaped system by considering the equivalent collision problem of three quasiparticles (Fig. 2). We label $V_{s w}^{(t \alpha)}$ and $V_{s w}^{(t \beta)}$ as the solitary wave speeds in the branches, referring to the branch with angles $\alpha$ and $\beta$, respectively. The speed of the reflected wave traveling back in the stem is indicated as $V_{s w}^{(r)}$, whereas, the incident wave speed is referred to as $V_{s w}^{(i)}$. The 
(a)

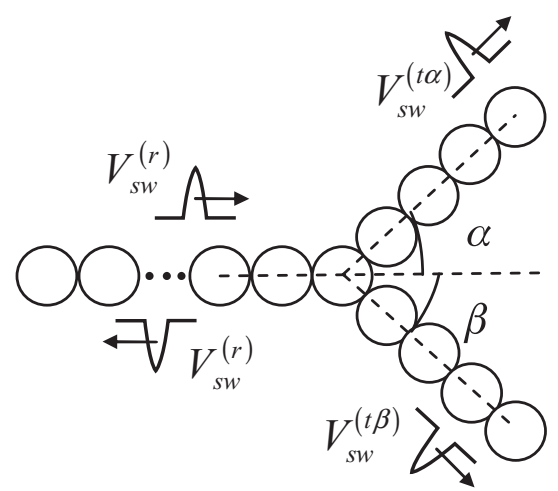

first quasiparticle, which represents the incident single solitary wave propagating in the main stem, travels with speed $V_{\text {eff }}^{(i)}$ and impacts two stationary quasiparticles, which represent the two branches without solitary wave propagation. After the impact, the first quasiparticle could be reflected with the speed $V_{\text {eff }}^{(r)}$ representing the reflected solitary wave propagation in the main stem, and the two branch quasiparticles move with the speeds $V_{\text {eff }}^{(t \alpha)}$ and $V_{\text {eff }}^{(t \beta)}$, corresponding to the transmitted solitary waves traveling in the branches. All the quasiparticles have the same mass $m_{\text {eff }}$ because all the particles in the main stem and the branches are identical [Eq. (1)]. From the conservation of momentum and energy before and after the impact, we obtain

$$
\begin{gathered}
m_{\mathrm{eff}} V_{\mathrm{eff}}^{(i)}=-m_{\mathrm{eff}} V_{\mathrm{eff}}^{(r)}+m_{\mathrm{eff}} V_{\mathrm{eff}}^{(t \alpha)} \cos \alpha+m_{\mathrm{eff}} V_{\mathrm{eff}}^{(t \beta)} \cos \beta, \\
0=m_{\mathrm{eff}} V_{\mathrm{eff}}^{(t \alpha)} \sin \alpha-m_{\mathrm{eff}} V_{\mathrm{eff}}^{(t \beta)} \sin \beta, \\
\frac{1}{2} m_{\mathrm{eff}}\left(V_{\mathrm{eff}}^{(i)}\right)^{2}=\frac{1}{2} m_{\mathrm{eff}}\left(V_{\mathrm{eff}}^{(r)}\right)^{2}+\frac{1}{2} m_{\mathrm{eff}}\left(V_{\mathrm{eff}}^{(t \alpha)}\right)^{2}+\frac{1}{2} m_{\mathrm{eff}}\left(V_{\mathrm{eff}}^{(t \beta)}\right)^{2},
\end{gathered}
$$

where $V_{\mathrm{eff}}^{(t \alpha)}$ and $V_{\mathrm{eff}}^{(t \beta)}$ are the speeds after impact of the quasiparticles of branch $\alpha$ and branch $\beta$, respectively. By solving Eqs. (12)-(14), we can obtain the ratios of solitary wave speeds as

$$
\begin{aligned}
& \frac{V_{s w}^{(r)}}{V_{s w}^{(i)}}=\left(\frac{V_{\mathrm{eff}}^{(r)}}{V_{\mathrm{eff}}^{(i)}}\right)^{1 / 5}=\left[\frac{\sin ^{2}(\alpha+\beta)-\sin ^{2} \alpha-\sin ^{2} \beta}{\sin ^{2}(\alpha+\beta)+\sin ^{2} \alpha+\sin ^{2} \beta}\right]^{1 / 5}, \\
& \frac{V_{s w}^{(t \alpha)}}{V_{s w}^{(i)}}=\left(\frac{V_{\mathrm{eff}}^{(t \alpha)}}{V_{\mathrm{eff}}^{(i)}}\right)^{1 / 5}=\left[\frac{2 \sin \beta \sin (\alpha+\beta)}{\sin ^{2}(\alpha+\beta)+\sin ^{2} \alpha+\sin ^{2} \beta}\right]^{1 / 5}, \\
& \frac{V_{s w}^{(t \beta)}}{V_{s w}^{(i)}}=\left(\frac{V_{\mathrm{eff}}^{(t \beta)}}{V_{\mathrm{eff}}^{(i)}}\right)^{1 / 5}=\left[\frac{2 \sin \alpha \sin (\alpha+\beta)}{\sin ^{2}(\alpha+\beta)+\sin ^{2} \alpha+\sin ^{2} \beta}\right]^{1 / 5}, \\
& \frac{V_{s w}^{(t \beta)}}{V_{s w}^{(t \alpha)}}=\left(\frac{V_{\mathrm{eff}}^{(t \beta)}}{V_{\mathrm{eff}}^{(t \alpha)}}\right)^{1 / 5}=\left[\frac{\sin \alpha}{\sin \beta}\right]^{1 / 5} .
\end{aligned}
$$

It should be noted that, when $\alpha+\beta>90^{\circ}$, the effective reflected wave speed becomes negative, implying that the quasiparticle representing the main stem continues to move forward after the collision, i.e., an incident solitary wave traveling in the main stem does not reflect at the $\mathrm{Y}$ junction and continues to travel forward, forcing the last particles in the main stem to separate from the chain. We restricted our experimental studies to the cases with $\alpha+\beta \leqslant 90^{\circ}$ to

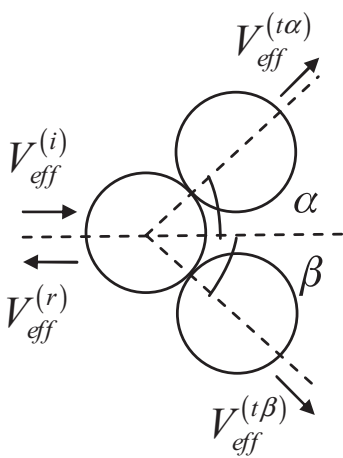

FIG. 2. (a) Schematic of the Yshaped system with the notation and direction of the solitary wave speeds in the system. (b) Schematic of the quasiparticles representation of the Yshaped system and its corresponding effective speeds. avoid complications caused by the chain's fragmentation. The amplitude of the solitary wave (maximum force) $F_{m}$ is related to the solitary wave speed as $F_{m} \sim V_{s w}^{6}$ [1]. Therefore, the ratio of the amplitude of the reflected wave to the amplitude of the incident wave and the ratio of the amplitude of the transmitted wave to the amplitude of the incident wave can be obtained as

$$
\begin{aligned}
\frac{F_{m}^{(r)}}{F_{m}^{(i)}} & =\left[\frac{\sin ^{2}(\alpha+\beta)-\sin ^{2} \alpha-\sin ^{2} \beta}{\sin ^{2}(\alpha+\beta)+\sin ^{2} \alpha+\sin ^{2} \beta}\right]^{6 / 5}, \\
\frac{F_{m}^{(t \alpha)}}{F_{m}^{(i)}} & =\left[\frac{2 \sin \beta \sin (\alpha+\beta)}{\sin ^{2}(\alpha+\beta)+\sin ^{2} \alpha+\sin ^{2} \beta}\right]^{6 / 5}, \\
\frac{F_{m}^{(t \beta)}}{F_{m}^{(i)}} & =\left[\frac{2 \sin \alpha \sin (\alpha+\beta)}{\sin ^{2}(\alpha+\beta)+\sin ^{2} \alpha+\sin ^{2} \beta}\right]^{6 / 5}, \\
\frac{F_{m}^{(t \beta)}}{F_{m}^{(t \alpha)}} & =\left(\frac{\sin \alpha}{\sin \beta}\right)^{6 / 5} .
\end{aligned}
$$

For the symmetric Y-shaped systems, the wave speed and amplitude ratios can be obtained from Eqs. (9) and (10) by setting $\alpha=\beta$.

\section{RESULTS AND DISCUSSION}

First, we investigated the propagation of single solitary waves in uniform Y-shaped systems, i.e., in systems composed of the same type of particles (stainless steel particles in this paper), for both symmetric and asymmetric configurations. For the symmetric uniform Y-shaped systems, we tested four different values of branch angles $\left(\alpha=\beta=30^{\circ}, 35^{\circ}, 40^{\circ}\right.$, and $\left.45^{\circ}\right)$. For the asymmetric uniform Y-shaped systems $(\alpha \neq$ $\beta$ ), we performed tests for cases of fixed $\alpha=30^{\circ}$ and $\beta=$ $30^{\circ}, 40^{\circ}, 50^{\circ}, 60^{\circ}$. The experimental results for the symmetric systems of $\alpha=\beta=30^{\circ}, 45^{\circ}$ are shown in Figs. 3(a)-3(c) presents the results for the asymmetric system of $\alpha=30^{\circ}$ and $\beta=60^{\circ}$. For these tests, the six instrumented particles were inserted in the following positions: 9 th and 12th particles in the main stem (counting from the impact side), and 5th and 8th particles in the two branches (counting from the interface), as pointed by the arrows in the corresponding curves in Fig. 3. In all the tests, the striker's impact velocity was $0.56 \mathrm{~m} / \mathrm{s}$. From these results, it is evident that the incident solitary wave traveling in the main stem crosses the Y-junction interface and splits into two single solitary waves traveling in the branches. For the symmetric systems, the transmitted solitary waves in the two branches are identical. This observation also was shown for the $30^{\circ}$ case in Ref. [26]. Unlike symmetric 

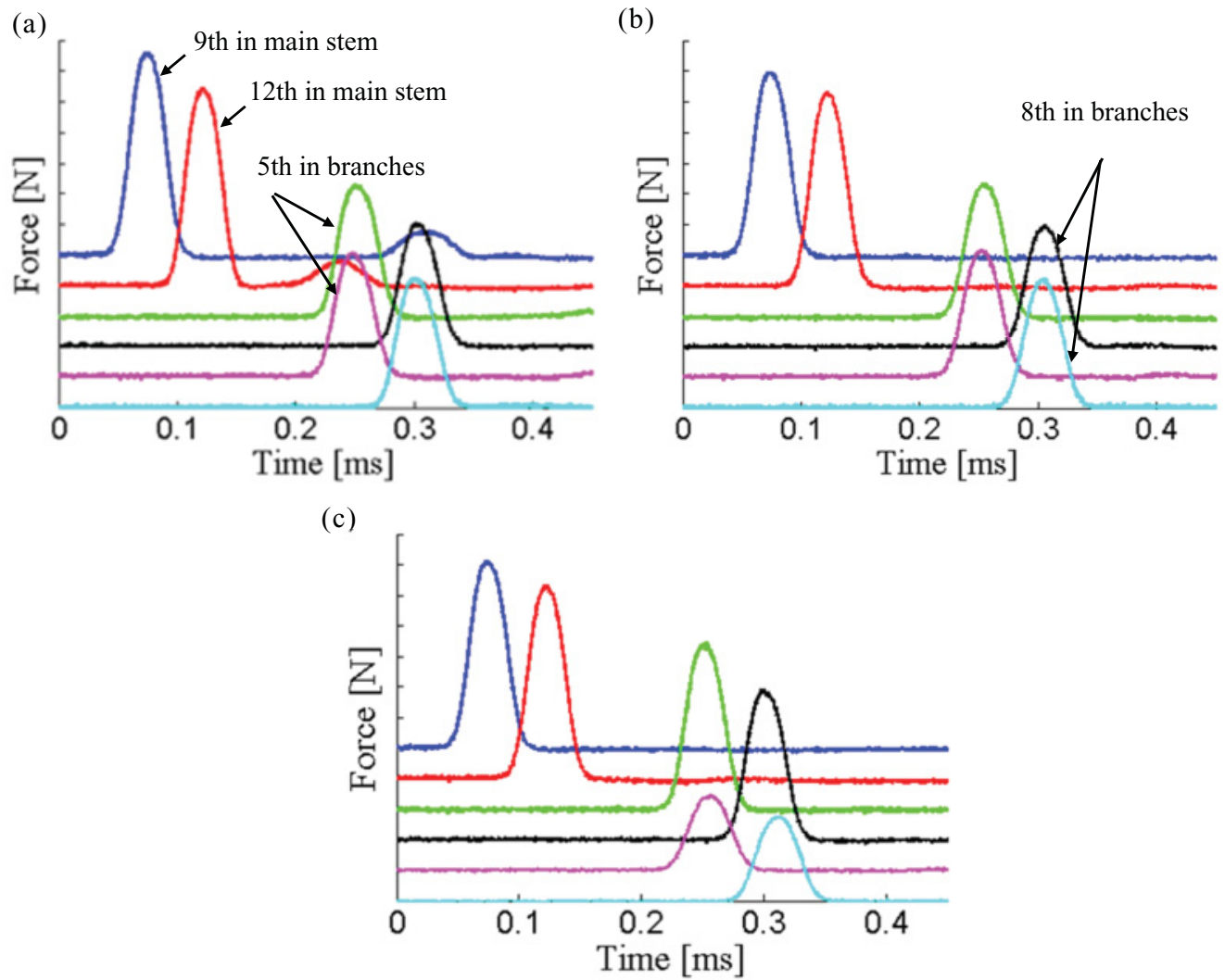

FIG. 3. (Color online) Experimental results for single solitary waves propagating in uniform Y-shaped systems composed of stainless steel beads. (a) $\alpha=\beta=30^{\circ}$. (b) $\alpha=\beta=45^{\circ}$. (c) $\alpha=30^{\circ}$ and $\beta=60^{\circ}$. The $y$-axes' scale for curves (a) and (b) is $10 \mathrm{~N}$ per division. The $y$-axes' scale for curve (c) is $15 \mathrm{~N}$ per division. The striker was dropped from the same height in all cases, and the striker velocity was $0.56 \mathrm{~m} / \mathrm{s}$.

Y-shaped systems, asymmetric systems support waves with different amplitudes and speeds in the two branches beyond the Y-shaped interface. The transmitted wave in the branch with the smaller branch angle ( $\alpha$ in this case) has a larger amplitude and a faster speed of propagation than the one in the other branch. In addition, a reflected solitary wave could be observed propagating backward from the $\mathrm{Y}$ junction in the main stem as shown in Fig. 3 (refer to the small amplitude pulses evident in the top two lines, reporting the signals detected in the main stem). The experimental results show that the amplitude and speed of the reflected waves decrease when the branch angles increase. In the case of $\alpha=\beta=45^{\circ}, \alpha=30^{\circ}$, and $\beta=$ $60^{\circ}$, no reflected wave in the main stem was observed in the experiments.

Next, we investigated the propagation of a train of solitary waves in both symmetric and asymmetric uniform Y-shaped systems composed of stainless steel beads. The train of solitary waves was generated in the systems by using a stainless steel particle of diameter $14.28 \mathrm{~mm}$ as the striker. The mass of the stainless steel striker is approximately three times larger than the mass of the particles in the systems. Experimental results are presented in Fig. 4. Similar to the behavior observed for the propagation of single solitary waves in the uniform symmetric Y-shaped systems, the solitary wave train also travels in the main stem and splits into two identical trains propagating in the two branches. In the asymmetric systems, the incident solitary wave train is transmitted unequally to the two branches. It should be noticed that a train of solitary waves reflected from the $\mathrm{Y}$ interface in the main stem formed when $\alpha+\beta<90^{\circ}$, but a quantitative analysis of the reflected waves was difficult because of their interaction with the incident train.

Experiments for symmetric nonuniform Y-shaped systems composed of chains of different materials were conducted next. We tested nonuniform systems composed of a chain of aluminum particles as the main stem and two chains of stainless steel particles as the two branches. An aluminum particle identical to the one composing the stem was used as the striker to create a single solitary wave in the main stem. The experimental results for these systems are shown in Fig. 5(a). In this case, the dynamic behavior of the system is similar to the corresponding uniform Y-shaped assemblies, except that, in the nonuniform system, the amplitude and speed of the reflected waves are higher, whereas, the amplitude and speed of the transmitted waves in the branches are smaller. Tests also were performed for other configurations of nonuniform systems in which the main stem and one branch were composed of stainless steel beads and the remaining branch was composed of aluminum beads. The experimental data for these systems are presented in Fig. 5(b). After the incident single solitary wave crosses the $\mathrm{Y}$ junction, it splits into one single solitary wave in the steel branch and a train of single solitary waves in the aluminum branch. It is interesting to note that, although the geometry of the systems is symmetric, due to the difference of materials in the two branches, the response of the systems is not symmetric. The amplitude of the transmitted wave in the branch of the heavier 

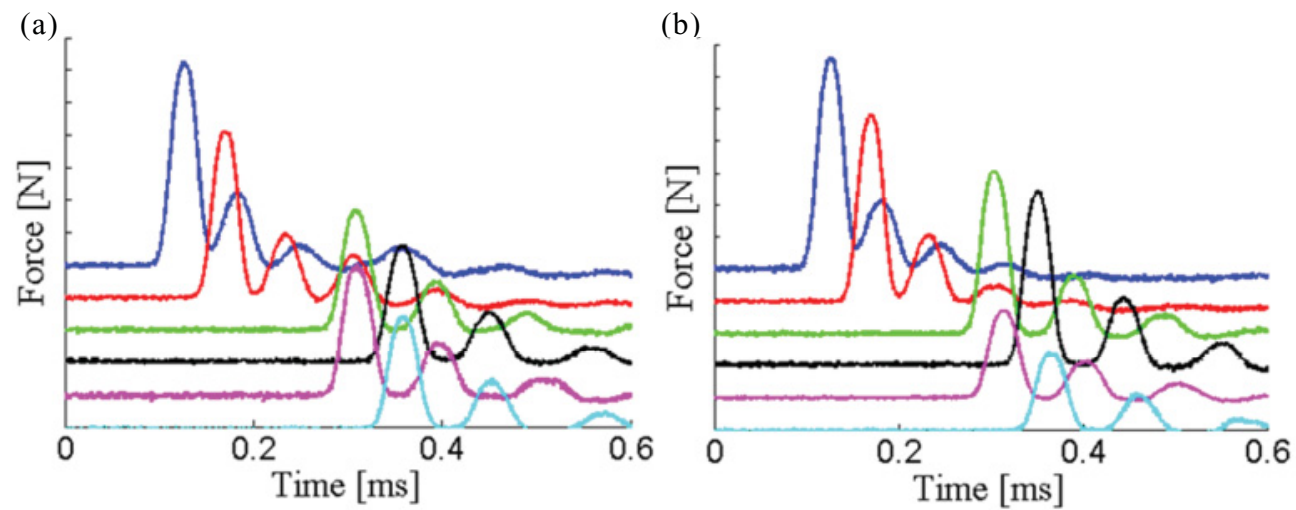

FIG. 4. (Color online) Experimental results for trains of solitary waves propagating in uniform Y-shaped systems composed of stainless steel beads. (a) $\alpha=\beta=30^{\circ}$. (b) $\alpha=30^{\circ}$ and $\beta=60^{\circ}$. The $y$-axes' scale for all the curves is $15 \mathrm{~N}$ per division. The striker was dropped from the same height in all cases, and the striker velocity was $0.45 \mathrm{~m} / \mathrm{s}$.

mass (stainless steel) is much larger than the amplitude of the wave in the branch of the lighter mass (aluminum). There was no reflected solitary wave observed in the range of branch angles we investigated.

Numerical simulations for wave propagation in the Yshaped system were performed using the 2D discrete element model summarized in the Appendix of the present paper and described in detail in Ref. [26].

We investigated the evolution of the solitary wave shape during the wave propagation in the systems. In particular, we chose the data from the single solitary wave propagation in the symmetric uniform Y-shaped system with $\alpha=\beta=$ $30^{\circ}$ to study. Figure 6 shows the solitary wave shape of the incident, transmitted, and reflected waves, obtained from the experiments, and their corresponding results obtained from discrete element simulations and theory. The theoretical wave shape is given by $F(t)=F_{m} \cos ^{6}\left(\frac{V_{s w} t}{\sqrt{10} R}\right)$, where $R$ is particle's radius, as discussed in Ref. [3]. We note that the measured wave shapes are very similar to the theoretical wave shapes also finding good agreement with the numerical simulation results. The spatial wavelength of the incident wave was calculated from experimental data to be $\sim 5.4$ particle size, compared with 5 particle size from the theory [1] and $\sim 5.8$ particle size from the discrete simulation. The values of spatial wavelength of the reflected wave in this case were $\sim 4.2, \sim 4.3$ particle sizes from the experiment and discrete simulation, respectively. For the transmitted wave in the branch, the experimental spatial length of the wave was $\sim 5.4$ particle size, and the numerical wavelength was $\sim 5.8$ particle size. We observed similar behaviors for the asymmetric uniform systems and nonuniform systems.

We studied the changes in wave speed and amplitude of a single solitary wave crossing the Y-junction interface in a uniform system as functions of the different branch angles. We calculated the ratios of wave speed and amplitude of the transmitted waves and reflected waves over the incident waves and compared the results obtained from the quasiparticle model, discrete element simulations, and experiments. The (a)

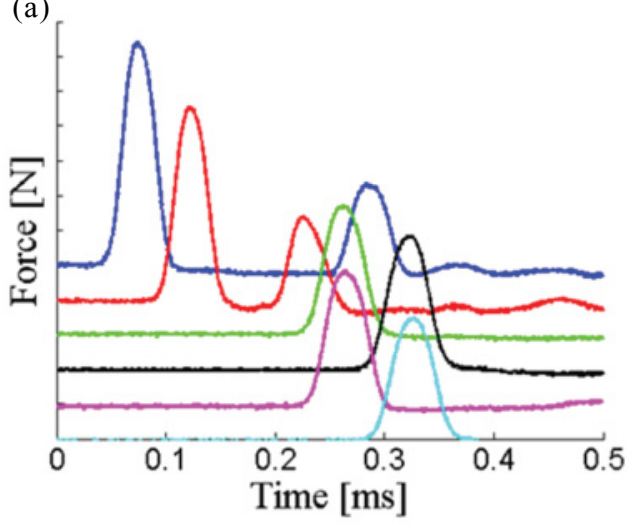

(b)

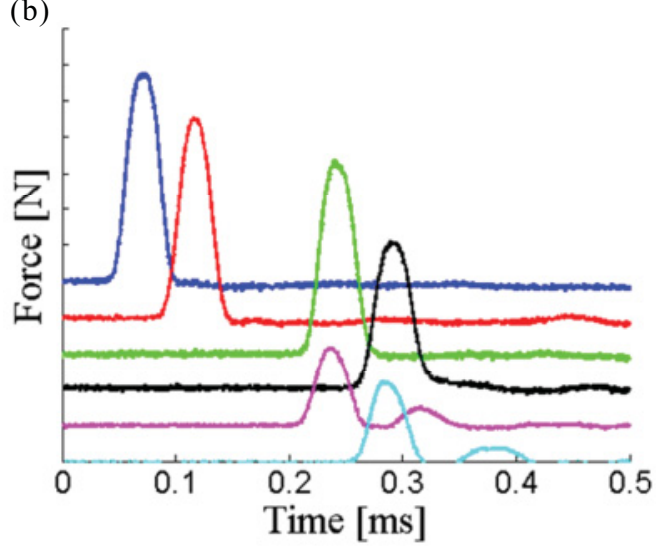

FIG. 5. (Color online) Experimental results for a single solitary wave propagating in nonuniform symmetric Y-shaped systems. (a) shows results obtained in a Y-shaped system in which the main stem was composed of aluminum beads and the two branches were composed of stainless steel beads for $\alpha=\beta=30^{\circ}$. The $y$-axes' scale for all the curves is $5 \mathrm{~N}$ per division, and the striker velocity was $0.71 \mathrm{~m} / \mathrm{s}$. (b) reports results obtained in a Y-shaped system in which the main stem and one branch are composed of stainless steel beads, whereas, the other branch is composed of aluminum beads for $\alpha=\beta=30^{\circ}$. The $y$-axes' scale for all the curves is $15 \mathrm{~N}$ per division, and the striker velocity was $0.67 \mathrm{~m} / \mathrm{s}$. 

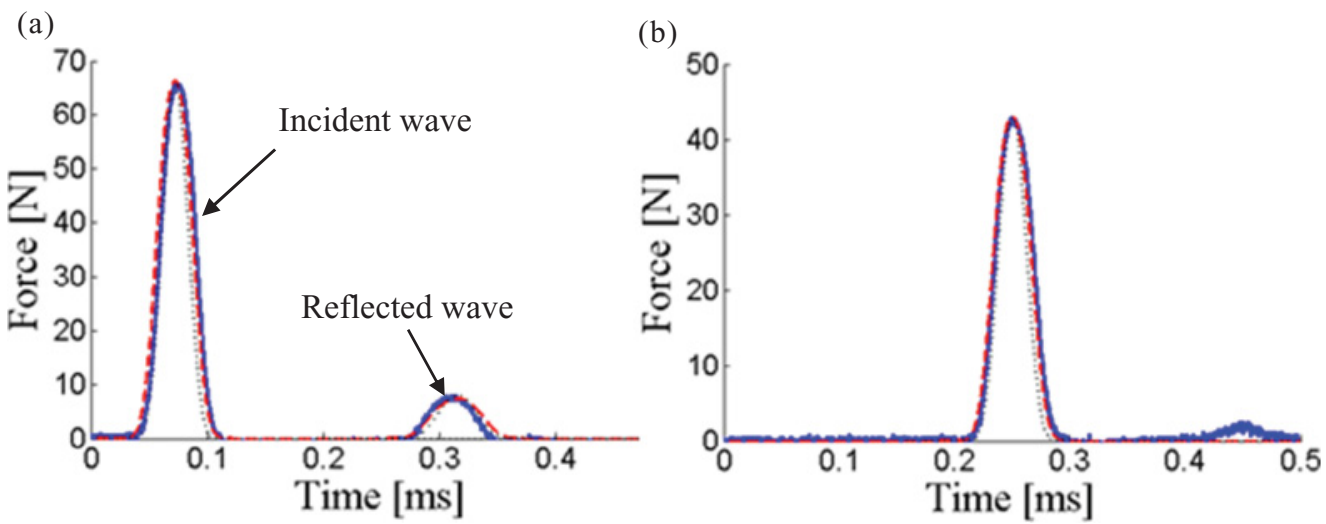

FIG. 6. (Color online) Comparison of the solitary wave shapes in uniform symmetric Y-shaped systems composed of stainless steel beads ( $\alpha=\beta=30^{\circ}$ ) for (a) incident and reflected solitary waves in the main stem, (b) transmitted solitary wave in the branch. The solid (blue) lines represent the experimental data. The dashed (red) lines are the discrete element simulation results. The dotted (black) lines are the theoretical wave shapes. The striker was dropped from the same height in all cases, and the striker velocity was $0.56 \mathrm{~m} / \mathrm{s}$.

results for the symmetric uniform systems are shown in Fig. 7, and the results for the asymmetric uniform systems are shown in Fig. 8. The error bars in the experimental data were calculated as the standard deviation of the data obtained from five measurements repeated with the same striker velocity for each set of branch angles. Results show good agreement among the quasiparticle model, the numerical simulations, and the experimental data. In the symmetric uniform Y-shaped systems (Fig. 7), for the same incident wave speed $V_{s w}^{(i)}$, the reflected wave speed $V_{s w}^{(r)}$ decreases as the branch angles increase, whereas, the transmitted wave speed $V_{s w}^{(t)}$ shows a reverse dependence on the branch angles. It should be pointed out that, in the case of $\alpha=\beta=45^{\circ}$, the quasiparticle model predicts no reflected wave traveling in the main stem. Differently, the numerical simulation predicts the presence of a reflected wave with very small amplitude ( $\sim 0.5 \%$ of the incident solitary wave amplitude), which was difficult to detect in the experiments. It is worth noting that the quasiparticle approach is based on a simplified modeling and, therefore, cannot capture all the complex dynamic interactions between particles in the system, especially at the $\mathrm{Y}$ junction.

Results obtained for the asymmetric uniform Y-shaped systems for the specific case when $\alpha$ is fixed at $30^{\circ}$ and $\beta$ varies from $30^{\circ}$ to $60^{\circ}$ are reported in Fig. 8. Now, due to the asymmetry of the two branches, the transmitted wave speeds $V_{s w}^{(t \alpha)}$ and $V_{s w}^{(t \beta)}$ in the branches do show different dependences on the branch angles. Figure 8(a) shows that, for the same incident wave speed $V_{s w}^{(i)}$ when angle $\beta$ increases $(\beta \geqslant \alpha)$, $V_{s w}^{(t \alpha)}$ increases while $V_{s w}^{(t \beta)}$ decreases. This implies that the ratio between transmitted wave speeds in the two branches represented by $V_{s w}^{(t \beta)} / V_{s w}^{(t \alpha)}$ also depends on the difference between two branch angles. The relation of reflected wave speed $V_{s w}^{(r)}$ to the branch angles in the asymmetric systems shown in Fig. 8(a) is similar to the corresponding one in the symmetric systems [Fig. 7(a)]. In the case where $\alpha=30^{\circ}$ and $\beta=60^{\circ}$, a reflected wave of very small amplitude was observed in numerical simulations, which was undetectable (a)

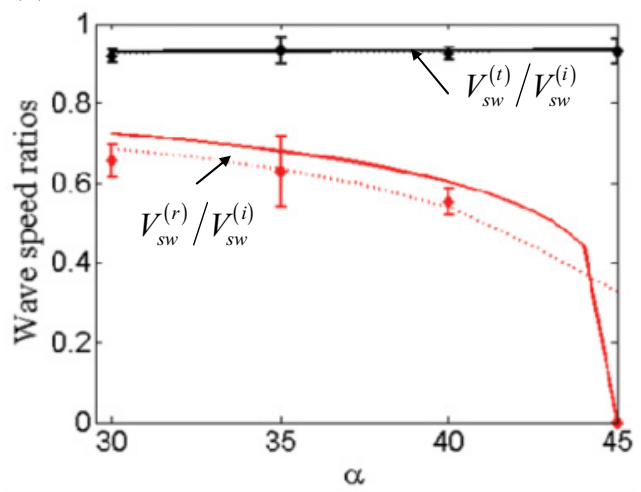

(b)

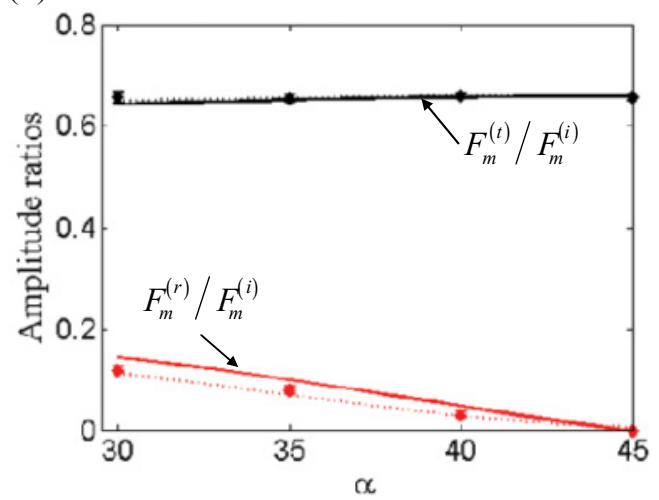

FIG. 7. (Color online) (a) Dependence of ratios of solitary wave speeds on the branch angles in a symmetric uniform Y-shaped system $(\alpha=\beta)$. (b) Dependence of ratios of solitary wave amplitudes on the branch angles in an asymmetric uniform Y-shaped system. The solid lines represent the results obtained from the quasiparticle model; the dashed lines represent results from the discrete element simulations. The ratios of the reflected wave to the incident wave are in red (bottom curves), the ratios of the transmitted wave to the incident wave are in black (top curves). Error bars denote the standard deviations obtained from five experimental measurements repeated with the same striker velocity for a fixed configuration of the system. 
(a)

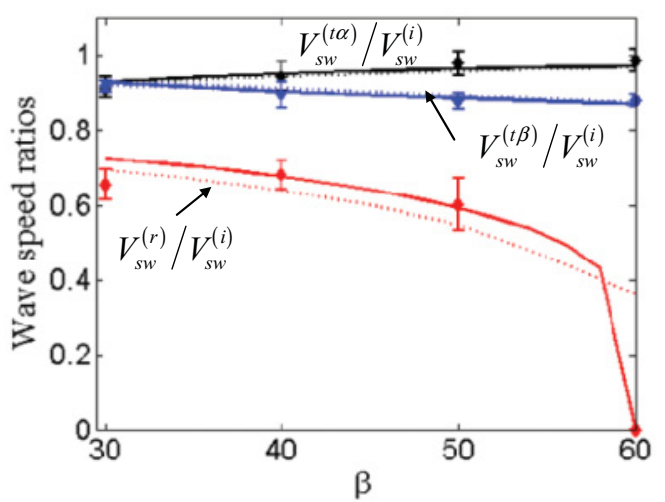

(b)

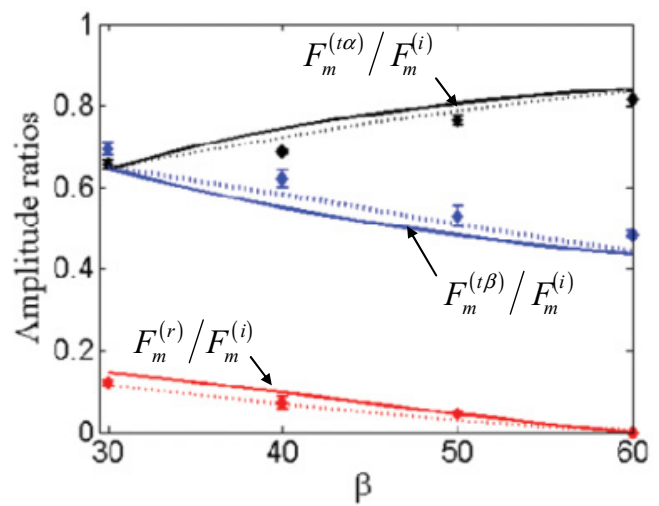

FIG. 8. (Color online) (a) Dependence of ratios of solitary wave speeds on the branch angle $\beta$ in a symmetric uniform Y-shaped system. (b) Dependence of ratios of solitary wave amplitudes on branch angle $\beta$ in an asymmetric uniform Y-shaped system. The solid lines represent the results from the quasiparticle model; the dashed lines represent the results from the discrete element simulations. The ratios of the reflected wave to the incident wave are in red (bottom curves), the ratios of the transmitted wave in branch $\alpha$ to the incident wave are in black (top curves), and the ratios of the transmitted wave in branch $\alpha$ to the incident wave are in blue (middle curves). Angle $\alpha$ was fixed at $30^{\circ}$. Error bars denote the standard deviations obtained from five experimental measurements repeated with the same striker velocity for a fixed configuration of the system.

in experiments. From Eqs. (11) and (16), the ratios of force amplitudes are shown to be proportional to the ratios of wave speeds, so we expect that the dependences of amplitude ratios on branch angles are similar to the ones of wave speed ratios. This is shown clearly in Figs. 7(b) and 8(b). The energy $E$ and the momentum $I$ carried by the single solitary wave (a)
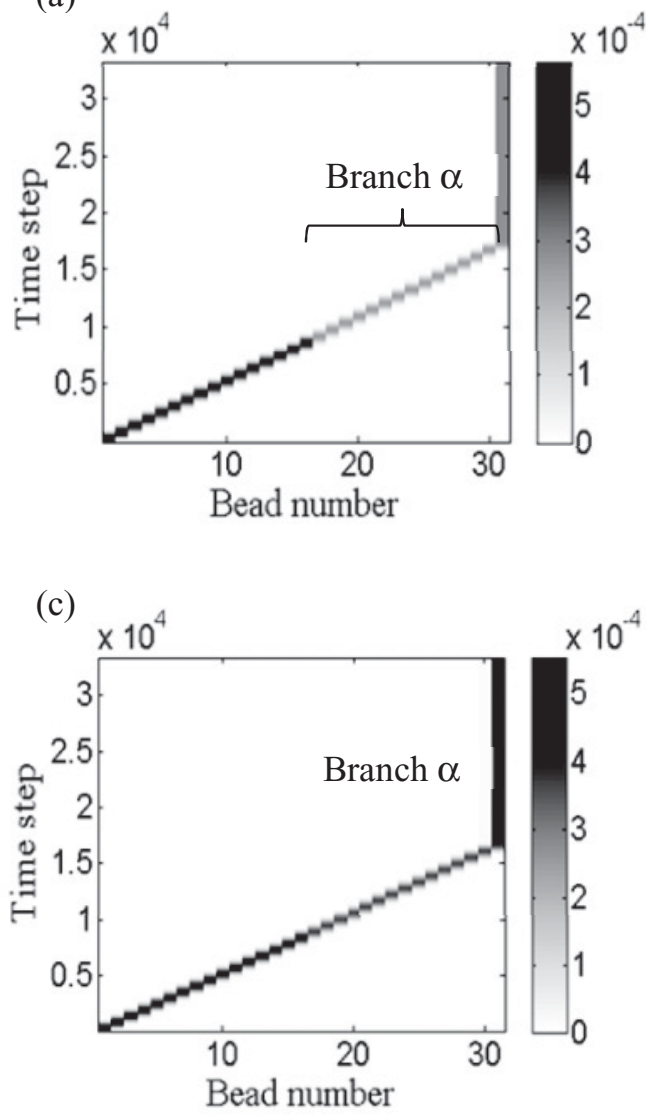

(b)

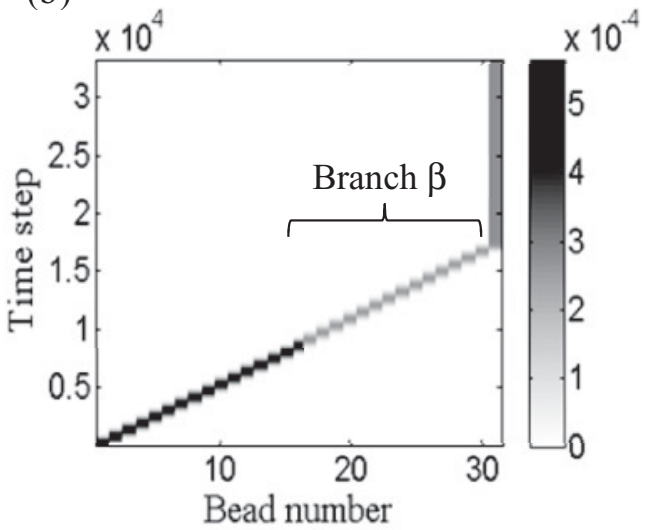

(d)

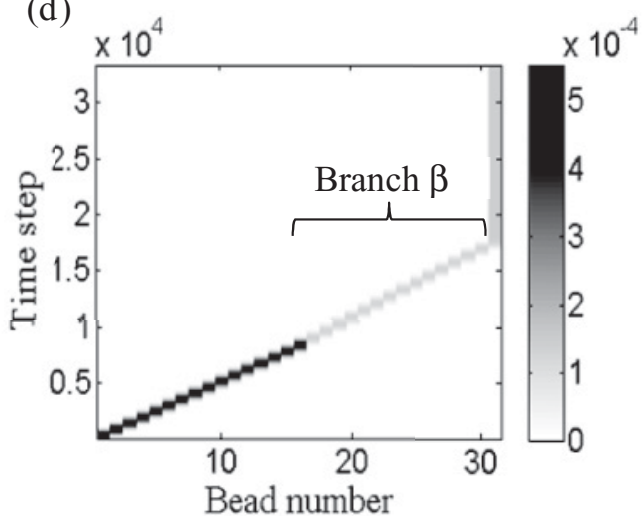

FIG. 9. (a) and (b) Energy density plots for single solitary wave propagation in symmetric uniform Y-shaped system $\alpha=\beta=45^{\circ}$. (c) and (d) Energy density plots for single solitary propagation in asymmetric uniform Y-shaped systems $\alpha=30^{\circ}$ and $\beta=60^{\circ}$. 
(a)

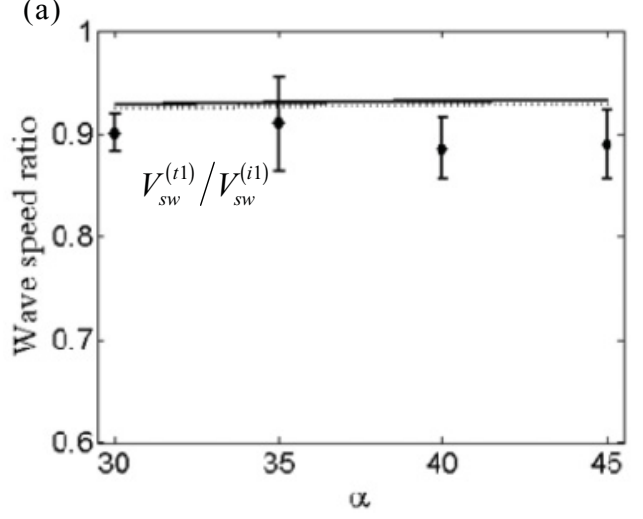

(b)

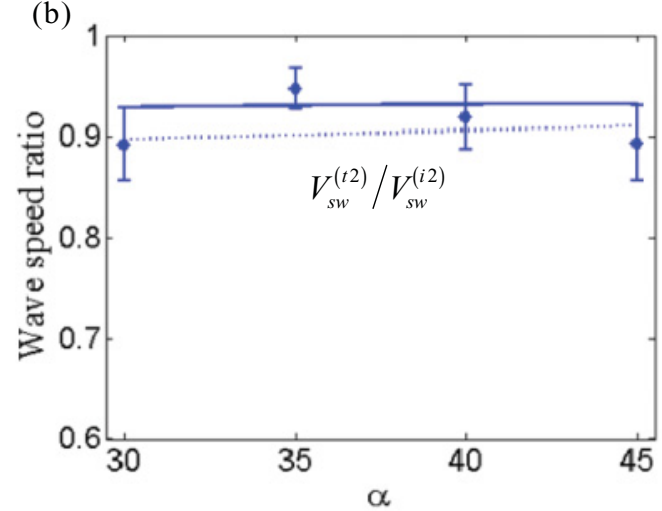

FIG. 10. (Color online) Dependence of the wave speed ratios on the branch angle in a symmetric $(\alpha=\beta)$ uniform Y-shaped system traversed by trains of solitary waves. (a) The wave speed ratios correspond to the speed of the first solitary wave in the transmitted train to the corresponding one in the incident train. (b) The wave speed ratios correspond to the speed of the second solitary wave in the train to the corresponding one in the incident train. The solid lines represent the results from the quasiparticle model; the dashed lines correspond to the results obtained with the discrete element simulations. Error bars denote the standard deviations obtained from five experimental measurements repeated with the same striker velocity for a fixed configuration of the system.

are proportional to the wave speed as $E \sim\left(V_{s w}\right)^{10}, I \sim$ $\left(V_{s w}\right)^{5}[1,16]$, hence, we also expect that the splitting of energy and momentum carried by the incident wave to two branches depends on the branch angles. Especially when utilizing the asymmetric Y-shaped systems, we can control the distribution of energy and the momentum carried by the incident wave transferred to each branch. To demonstrate the capability of distributing different amounts of energy to the branches of the Y-shaped system, here, we present the energy density plots obtained from the discrete element simulations (Fig. 9). The energy density plots illustrate the total energy (the sum of potential energy and kinetic energy) of each bead, at each time step, as the solitary wave propagates in the system. In the plots, the abscissa shows the bead number starting from the striker as bead number 1 , the last bead of the main stem is bead number 16, and the last bead of each branch is labeled as bead number 31 . The color map represents the magnitude of the energy. From Fig. 9, it clearly is observed that the energy carried by the incident solitary wave in the symmetric uniform Y-shaped system is split equally to the branches, whereas, in the asymmetric system, more energy is transferred to the branch with a smaller branch angle. The above results show that, by changing the respective angles $\alpha$ and $\beta$, stress waves can be redirected along different paths, and their amplitude, speed, and energy can be determined fully based on the branch angles. This interesting property of the asymmetric Y-shaped system could be used for the design of acoustic delay line systems or stress mitigation engineered materials.

Next, we study the propagation of a train of solitary waves in symmetric and asymmetric uniform systems. Figures 10 and 11 show the dependence on the branch angles of the wave speed ratios of the first and second solitary waves in
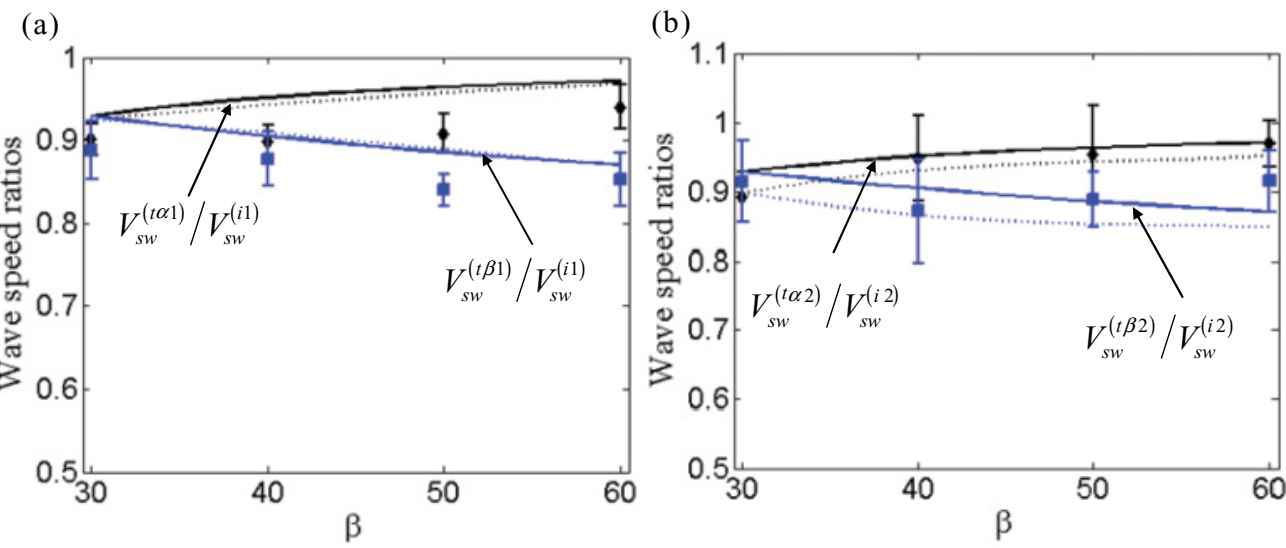

FIG. 11. (Color online) Dependence of the wave speed ratios on the branch angle in an asymmetric uniform Y-shaped system traversed by trains of solitary waves. (a) The wave speed ratios correspond to the speed of the first solitary wave in the transmitted train to the corresponding one in the incident train. (b) The wave speed ratio corresponds to the speed of the second solitary wave in the transmitted train to the corresponding one in the incident train. The solid lines represent the results from the quasiparticle model; the dashed lines are the discrete element simulation results. Error bars denote the standard deviations obtained from five experimental measurements repeated with the same striker velocity for a fixed configuration of the system. 
(a)

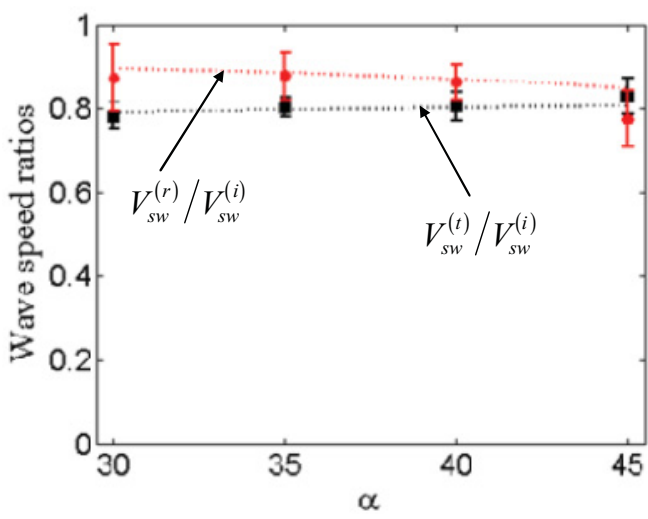

(b)

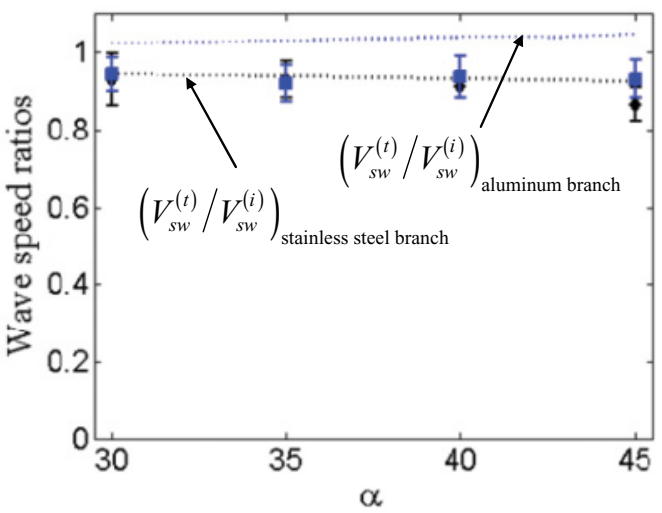

FIG. 12. (Color online) Dependence of the wave speed ratios on the branch angle in a symmetric nonuniform Y-shaped system. (a) System composed of a main stem filled with aluminum particles and branches filled with stainless steel particles. (b) System composed of a main stem and a branch filled with stainless steel particles and the other branch filled with aluminum particles. The dashed lines report results obtained with the discrete element simulations. Error bars denote the standard deviations obtained from five experimental measurements repeated with the same striker velocity for a fixed configuration of the system.

the train, respectively, obtained from experiments, discrete element simulations, and the quasiparticle model. The results presented in the above figures highlight the fact that the first solitary wave exhibits a correlation between wave speed ratios and branch angle that is similar to that characterizing a single solitary wave [Figs. 10(a) and 11(a)], whereas, the second solitary wave shows smaller wave speed ratios. Such a difference between the first and the second solitary wave in the train is most likely due to the reduced number of particles forming the current experimental setup, which does not allow the (incident and transmitted) secondary solitary waves to reach a steady state. In order to clarify the matter, we performed discrete numerical simulations for systems composed of a larger number of particles (50 particles in each branch with sensors in two branches placed far from the Y junction). Our investigations on dense particle systems showed that the dependence of secondary wave speed ratios on the branch angles closely follows the one observed for a single solitary wave, which confirms that a train of single solitary waves actually is composed of independent single solitary waves $[1,16]$.

We study nonuniform symmetric systems and focus our attention on the effects induced by variations in the branch angles on the propagation of solitary waves. The first nonuniform system studied consists of a main stem composed of aluminum particles and two branches composed of stainless steel particles. The dependence of the solitary wave speed on the branch angle for this system is shown in Fig. 12(a). For the same incident wave speed $V_{s w}^{(i)}$, the transmitted wave speed $V_{s w}^{(t)}$ increases, and the reflected wave speed $V_{s w}^{(r)}$ decreases as the branch angles increase. Comparing this nonuniform system under examination with a uniform system of stainless steel beads, we observe that the first one exhibits a smaller $V_{s w}^{(t)} / V_{s w}^{(i)}$ ratio and a considerably higher $V_{s w}^{(r)} / V_{s w}^{(i)}$ ratio, respectively, over the second one. This can be explained by the fact that the mass of the stainless steel particles in the branches is larger than the mass of the aluminum particles in the main stem. The second nonuniform system we studied consists of the main stem, one branch composed of stainless steel particles, and the other branch composed of aluminum particles. The dependence of the solitary wave speed on the branch angle for this system is shown in Fig. 12(b) for each of the two branches. In this case, we only study the properties of the first wave in the train traversing the branch with aluminum beads. From discrete element simulations, we observe that, in the stainless steel branch, the wave speed decreases as the angles increase, whereas, in the aluminum branch, the wave speed increases as the angles increase.

\section{CONCLUSIONS}

We studied the dynamics of single solitary waves and trains of solitary waves propagating in both uniform and nonuniform Y-shaped systems with different branching geometries and materials. We experimentally tested the response of systems assembled in support guides with different geometries and compared the results with numerical simulations obtained through a discrete element model. We also employed a quasiparticle model to obtain analytic solutions. We studied the changes in wave speeds and amplitudes before and after the waves cross the $\mathrm{Y}$ junction as a function of the branch angles. We found excellent agreement among numerical, analytic, and experimental data. Asymmetric Y-shaped systems showed a special ability to redirect stress waves along different directions. The possibility to distribute different amounts of energy in the two branches as a function of the branch angles could be exploited in a variety of practical applications, including impact protecting systems, energy harvesting systems, and delay lines.

\section{ACKNOWLEDGMENTS}

Support for this work was received from the Army Research Office (MURI Grant No. US ARO W911NF-09-1-0436 and Proposal No. 54272-EG). 


\section{APPENDIX: DISCRETE ELEMENT MODELING}

We formulate a discrete element modeling of the Y-shaped system by describing the particles as point masses connected by nonlinear springs, which reproduce the normal Hertzian contact law between spheres. Such a simplified nondissipative modeling neglects irreversible phenomena, such as, e.g., friction and plasticity, and is aimed to capture the main dynamical features of a 2D granular system composed of a small number of grains. The trajectory of the generic particle (say, the $i$ th one) is determined through numerical integration of the following equation of motion:

$$
m_{i} \dot{\mathbf{V}}_{i}=-\sum_{j \neq i} \alpha_{i j}\left(\delta_{i j}^{+}\right)^{3 / 2} \mathbf{n}_{i j}
$$

where $\dot{\mathbf{V}}_{i}$ is the particle velocity vector, the dot denotes the time derivative, $m_{i}$ is the particle mass, the summation on the right hand side is extended to all the particles in the systems different from the $i$ th one, $\delta_{i j}^{+}$is the positive part of the normal penetration displacement $\delta_{i j}$ between particles $i$ and $j$ [26], $\mathbf{n}_{i j}$ is the unit vector in the direction connecting the centers of mass of particles $i$ and $j$, outwardly oriented with respect to particle $i$, and $\alpha_{i j}$ is the following scalar coefficient:

$$
\alpha_{i j}=\frac{4 E_{i} E_{j} \sqrt{\frac{r_{i} r_{j}}{r_{i}+r_{j}}}}{3 E_{i}\left(1-v_{j}^{2}\right)+3 E_{j}\left(1-v_{i}^{2}\right)},
$$

with $E_{i}, v_{i}$, and $r_{i}$ denoting the Young modulus, the Poisson ratio, and the radius of the $i$ th particle, respectively. The walls of the Y-shaped guides are modeled as particles with infinite radii, fixed coordinates, $\mathbf{n}_{i j}$ always coincident with the outward normal to the guide (for any $j$ ), and $E_{i}$ and $v_{i}$ correspondent to the elastic properties of the polycarbonate in Table I.

We integrate Eq. (A1) over time, for each particle in the system, through a fourth-order Runge-Kutta method with time step $\Delta t=10^{-8} \mathrm{~s}$. Such a choice leads us to obtain integration errors lower than $10^{-5} \%$ in terms of total energy conservation.
[1] V. F. Nesterenko, Dynamics of Heterogeneous Materials (Springer-Verlag, New York, 2001).

[2] V. F. Nesterenko, Prikl. Mekh. Tekh. Fiz. 24, 136 (1983) [J. Appl. Mech. Tech. Phys. 24, 733 (1984)].

[3] C. Coste, E. Falcon, S. Fauve, Phys. Rev. E 56, 6104 (1997).

[4] S. Sen, M. Manciu, and J. D. Wright, Phys. Rev. E 57, 2386 (1998).

[5] C. Coste and B. Gilles, Eur. Phys. J. B 7, 155 (1999).

[6] J. Hong and A. Xu, Appl. Phys. Lett. 81, 4868 (2002).

[7] A. Rosas and K. Lindenberg, Phys. Rev. E 69, 016615 (2004).

[8] J. Hong, Phys. Rev. Lett. 94, 108001 (2005).

[9] R. L. Doney and S. Sen, Phys. Rev. E 72, 041304 (2005).

[10] V. F. Nesterenko, C. Daraio, E. B. Herbold, and S. Jin, Phys. Rev. Lett. 95, 158702 (2005).

[11] S. Job, F. Melo, A. Sokolow, and S. Sen, Phys. Rev. Lett. 94, 178002 (2005).

[12] C. Daraio, V. F. Nesterenko, E. B. Herbold, and S. Jin, Phys. Rev. E 72, 016603 (2005).

[13] C. Daraio, V. F. Nesterenko, E. B. Herbold, and S. Jin, Phys. Rev. E 73, 026610 (2006).

[14] C. Daraio and V. F. Nesterenko, Phys. Rev. E 73, 026612 (2006).

[15] C. Daraio, V. F. Nesterenko, E. B. Herbold, and S. Jin, Phys. Rev. Lett. 96, 058002 (2006).

[16] S. Job, F. Melo, A. Sokolow, and S. Sen, Granular Matter 10, 13 (2007).

[17] S. Sen, J. Hong, J. Bang, E. Avalos, and R. Doney, Phys. Rep. 462, 21 (2008).
[18] M. A. Porter, C. Daraio, E. B. Herbold, I. Szelengowicz, and P. G. Kevrekidis, Phys. Rev. E 77, 015601 (2008).

[19] M. A. Porter, C. Daraio, E. B. Herbold, I. Szelengowicz, and P. G. Kevrekidis, Physica D 238, 666 (2009).

[20] F. Fraternali, M. A. Porter, and C. Daraio, Mech. Adv. Mater. Struct. 17, 1 (2010).

[21] D. Khatri, C. Daraio, and P. Rizzo, Proc. SPIE 6934, 69340U (2008).

[22] A. Spadoni and C. Daraio, Proc. Natl. Acad. Sci. USA 107, 7230 (2010).

[23] Y. Zhu, A. Shukla, and M. H. Sadd, J. Mech. Phys. Solids 44, 1283 (1996).

[24] A. Shukla, C. Y. Zhu, and M. H. Sadd, J. Strain Anal. Eng. Des. 23, 121 (1988).

[25] C. Daraio and V. F. Nesterenko, in APS-Shock Compression of Condensed Matter, edited by M. Elert, M. D. Furnish, R. Chau, N. Holmes, and J. Nguyen, AIP Conf. Proc., No. CP955 (AIP, New York, 2007), p. 1419.

[26] C. Daraio, D. Ngo, V. F. Nesterenko, and F. Fraternali, Phys. Rev. E 82, 036603 (2010).

[27] D. Ngo, F. Fraternali, and C. Daraio, Proceedings of the ASME 2010 International Mechanical Engineering Congress \& Exposition, IMECE2010-39699, Vancouver, Canada (2010).

[28] N. Boechler, G. Theocharis, and C. Daraio, Nat. Mater. 10, 665 (2011).

[29] [http://www.efunda.com; http://asm.matweb.com]. 